$32^{\text {nd }}$ European Modeling \& Simulation Symposium

$17^{\text {th }}$ International Multidisciplinary Modeling \& Simulation Multiconference

ISSN 2724-0029 ISBN 978-88-85741-44-7 C 2020 The Authors.

doi: 10.46354/i3m.2020.emss.061

\title{
Artificial Intelligence to Support Retail Sales Optimization
}

\section{Agostino G. Bruzzone ${ }^{1,2}$ *, Kirill Sinelshchikov ${ }^{2}$, Marina Massei ${ }^{1,2}$, Wolfhard Schmidt ${ }^{3}$}

${ }^{1}$ SIM4Future, Simulation Team, via Trento 34, 16145, Genova, Italy

${ }^{2}$ Simulation Team, DIME, University of Genoa, via Opera Pia 15, 16145 Genova, Italy

${ }^{3}$ Simulation Team, via Magliotto, 17100, Savona, Italy

*Corresponding author. Email address: agostino.bruzzone@sim4future.com

\begin{abstract}
Presented study focuses on utilization of Artificial Intelligence (AI) in order to support data integration, sales forecasting and process optimization in retail. In particular, use of Artificial Neural Networks (ANN) and Genetic Algorithms (GA) in order to support decision makers from sales departments has evaluated.
\end{abstract}

Keywords: Genetic Algorithms, Artificial Neural Networks, Sales Forecasting, Retail

\section{Introduction}

For many decades, acquisitions and sales in retail were managed based on personal experience. Indeed, while it was possible to have data about average sales in time-period and generic trends, fine grained evaluation of correlations was unattainable for simple algorithms and was done mostly by intuition of decision makers - Biological Neural Networks. In last decades advances in Artificial Intelligence (AI), in particular regarding usability, documentation and support, allowed to the new generation of decision makers to take benefit from these systems in order to facilitate, improve and speed-up various business related procedures.

One of most interesting from economical point of view goals in retail could be identification of set of best products to put into promotion. Indeed, advertising allows to present shops as more convenient respect to competitors and to drive additional sales. In the same time, promotion could be used in order to familiarize clientele with new line of products. Obviously, there are numerous factors to be taken into account, such as initial popularity of the goods, seasonality of their consumption (e.g. salad is best sold in summer, while chocolate performs better during cold seasons), actions of competitors respect same or similar product groups, promotion companies started by the producers (e.g. TV advertisement, internet marketing company), stock availability respect to expected sales, overall trends over year (e.g. preference to ecological and "bio" alternatives) etc (Ansari \& Joloudar 2011). Similarly, some particular products are sold only during specific time-periods and their presence in promotion out of it would not have any sense; indeed, each culture could be characterized by a set of holidays and corresponding traditions. For instance, in many Christian Countries chocolate eggs are sold right before the Easter, while other typical spikes in consumption of particular products could be related to other holidays, such as Halloween, Oktoberfest or Christmas. Similarly, sales depend on much more local or less predictable factors, such as weather conditions (Verstraete et al., 2019).

(C) 2020 The Authors. This article is an open access article distributed under the terms and conditions of the Creative Commons Attribution (CC BY-NC-ND) license (https://creativecommons.org/licenses/by-nc-nd/4.0/). 
Indeed, sales of umbrellas surge during the rain, especially in cases when it starts unexpectedly so the people is not prepared to it; as another example, even a small shop could be responsible for significant sales of anti-limescale detergent in case of a poor water quality in a zone. Apart from this, the promotion strategy itself must be taken into consideration, especially regarding discount and position in the offer, for instance if the product should be used as one of most advertised assets or keep in the list as one among many others (d'Astous \& Landreville, 2003). Considering this, it is important to introduce in promotion proper products in right moments of time. In order to achieve this goal, it is essential to be able to answer to the main question: "How much of product $\mathrm{X}$ would be sold in conditions Y?".In order to get the answer, it is possible to benefit from modern technologies and methodologies, for example, by employing Artificial Neural Networks (ANN) in order to forecast sales based on different combinations of input parameters. Indeed, in case of availability of samples of sufficient quantity and quality it is possible to train the Artificial Intelligence (AI) to recognize influence of numerous factors on results of sales of a product.

Therefore a critical aspect to solve this problem in real problems is to properly involve the decision makers in the loop and give them possibility to control the optimization process in order to develop an effective accreditation of current situation (Bruzzone et al., 2007).

Obviously, capability to forecast sales is only the first step in research of the best combination for advertising company. Indeed, based on statistical data it is possible to obtain number of stock keeping units (SKU) in a typical grocery store, which usually falls in range between 40000 and 60000 (Pak et al. 2020). Considering this, and the fact that one product could have tens of SKU (e.g. different tastes of biscuits of the same brand, formats of pasta etc.), it is evident that in order to evaluate an offer it is required to check performance of thousands of products. Furthermore, other factors such as price rise further complexity of computation; indeed, each product could have several fixed acceptable discount values, which could be for example $10 \%, 20 \%$ and $30 \%$ of its normal price. Considering all these factors and typical necessity to plan advertisement ahead for at least several weeks, it is evident that number of different combination of SKUs and their discounts could exceed several millions, making brute force check poorly suitable for this goal.

Other parameters such as mutual exclusion of some products from offer and minimal time-periods between putting same product in advertisement are to be considered. Indeed, it would be not effective to put contemporary in the promotion multiple products belonging to the same category, for instance belonging to competitive brands. In the same time, by definition an offer cannot last forever and should appear with some time intervals in between. Finally yet importantly, promotion of one single product impacts also sales of others, hence, even a solution which looks promising in offer could diminish positive impact of offer on overall sales.

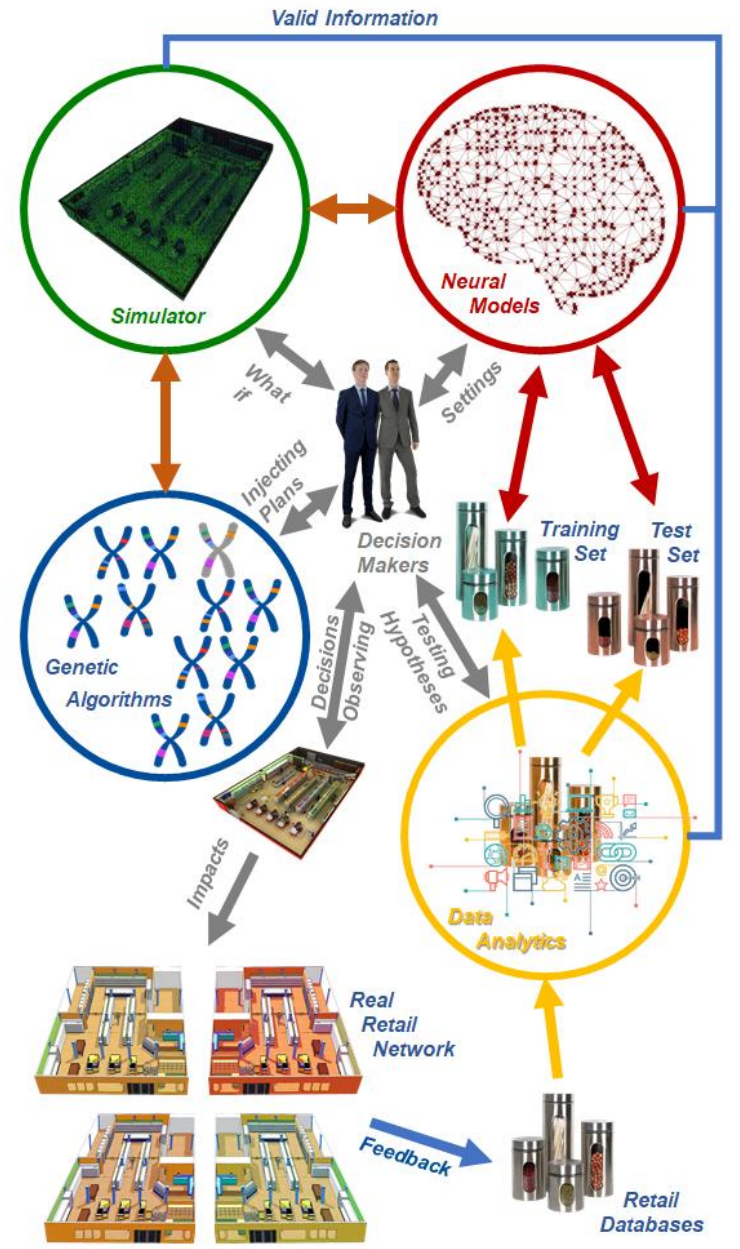

Figure 1 General Architecture based on Strategic Engineering principia

In order to overcome this limitation, the authors propose an automated decision support solution, capable to generate various efficient advertising proposals taking into account boundary conditions and mutual influences of products. In particular, it is decided to employ a Genetic Algorithm (GA) in order to optimize research for an optimal or near-optimal solution. Genetic Algorithms are belonging to the family of evolutionary algorithms, which are inspired by concept of natural selection and evolution (Darwin, 1859). Indeed, GAs employ such evolutionary mechanisms as mutations (stochastic modification of a solution) and crossover (combination of two parent solutions) in order to create next generation of known population of possible solutions; in this case, members of the next generation are chosen based on their performance score, which in case of interest corresponds to the value of sales.

Thanks to this approach, every new generation of solutions, performs not worse than the previous one, 
while during evolution the overall score grows, making it possible to find always better solutions in terms of expected sales.

\section{State of the art}

There are different ways in which Artificial Intelligence (AI) could be used to optimize retail. For instance, chatbots are now often used in e-commerce in order to help customers with at least not too complex tasks, vice versa, some experimentation are done to evaluate efficiency of intelligent robotic systems for ins-store assistance in physical shops, as a kind of counterpart to virtual chatbots (Bogue, 2019). These systems are indeed capable to help customers in most of the cases and lead to improvement of satisfaction (Brill et al., 2019), which is true even for luxury services (Chung et al., 2018).

In some physical stores and trade centers clients' smartphones are used as smart assistants, which allows to reduce costs but still to provide service and support to the customers (Setiabudi \& Tjahyana, 2011). In many cases visual search and clustering, another typical problems addressed by machine learning, could be helpful to find similar products in order to demonstrate relevant recommendations to the clients (Wallace et al., 2003). Often the systems are able to identify hidden behavioral patterns, hence, to propose distinct but relevant solutions (Kengpol \&Wangananon, 2006).

Other systems are used to evaluate customer satisfaction and predict behavior, which is especially useful to support introduction of proper solutions and modifications in retail; in some situations the data is taken not only from stores but from posts in social networks (Kumar \& Zymbler, 2019). Finally, other kind of intelligent systems is used to perform price analysis in order to predict future trends as well as to adjust existing prices, to reduce unwanted out-ofstock cases and leftovers (Ferreira et al., 2016). (Bruzzone\&Longo, 2010)

\section{Strategic Engineering \& Retail Optimization}

The proposed architecture is based on the principia that are peculiar of the Strategic Engineering, a new discipline that emphasize the importance to combine M\&S (Modeling \& Simulation), Data Analytics and AI (Artificial Intelligence) in closed loop with the real systems and directly interconnected with decision makers as proposed in figure 1 (Bruzzone et al., 2018).

In this case, the top executives are at the center of an architecture where data, information and models are continuously updated and affected by the boundary condition changes, reactions of competitors and new decisions.

In this case the real retail network generates many data that are extended by other sources including weather forecasts, economic trends, competitor evolution, supply chain, etc (Bruzzone, Massei \& Bocca 2009). These big data are obviously including many inconsistencies and potential errors and it is necessary to apply Data Analytics devoted specifically to cross relate the data in order to filter, process, elaborate and fuse data to obtain useful and valid information. From this point of view, it is generated a consistent database that it is available to managers to check their hypothesis directly; this aspect is very important even considering that the mix of products distributed by the retail network is subjected to changes (e.g. seasonality, obsolescence, new products) that could require to develop the capability to define a bidirectional connection between items and meta categories (Bruzzone, Bocca, Pierfederici \& Massei 2009). At the same time these checked data sources are used to create training and test set to apply Machine Learning based on Artificial Neural Networks for developing Neural Models able to support forecasts on retail performance of the different products and marketing alternatives. The decision makers have a limited capability to interact with the ANN considering that usually they could just act on high level parameters devoted to their tuning (e.g. time horizon of forecasts, categories to be investigated, different point of sales to be included). The Neural Models are used by the Simulator in order to evaluate specific decisions that are affected by transversal factors; these models are available for simple what if analysis by managers as well as for more effective evaluations based on techniques such as DOE (Design of Experiments). The Genetic Algorithms (GAs) operate as an Optimizer by generating a population of possible solutions respect retail strategies that are evaluated quantitatively by Simulation using Neural Models to find most promising decisions. The top executive could inject in GAs their own proposal that could be used to fertilize the population with additional genetic material and compete with other alternatives proposed by the Optimizer. The best proposals are evaluated by decision makers that turn them in real actions that affect the retail network and generate soon feedback to guarantee a continuous and persistent machine learning process. It is important to outline that to succeed in this plan it is fundamental to use highly qualified experts not only in designing the details of the architecture and developing the different components, but also to engage the decision makers and guarantee the accreditation of the models and of the new decision processes; it is evident that this results is strongly related with Verification and Validation procedures based on experimentation of the solution on the field and extensive testing (Bruzzone, Bocca \& Poggi, 2009).

\section{The Forecasting and Optimization System}

In order to optimize advertisement of products, it is possible to utilize different techniques, however, in the particular case it was decided to adapt genetic algorithm in order to find best solution based on a set of initial hypothetical combinations. Indeed, this 
solution seems particularly suitable considering the fact that in many cases it is possible to reutilize old promotions as starting point for the development of the new ones, in the same time, an expert could manually include list of products and promotional parameters that seems promising. Hence, GA could benefit from presence of already known wellperformant solutions, facilitating the convergence of the algorithm. Indeed, at the first step the system loads a list of proposed advertisements and analyzes all products in order to have a rough estimation of their possible performance. Indeed, since the beginning the system assigns weights to all known products in order to generate reasonable combinations instead of purely stochastic ones. At the next step, the GA starts analysis of proposed combinations of products by forecasting expected sales, combining proposals one with another and adding minor stochastic modifications in order to create the next set of advertising strategies.

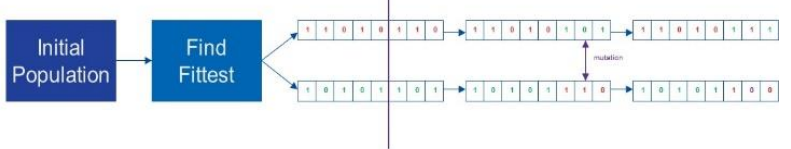

Figure 2 Optimization \& Genetic Algorithms

In the same time, the system guarantees the constraints are satisfied, for instance, that products have reasonable discounts, offers are repeated with acceptable frequencies and similar products in the same offer are avoided.

The software takes care of reading, validation and integration of data provided in different files, training and control of the neural network as well as of the search of the best solution.

Indeed, the first typical step in development of data analytics and forecasting systems is preparation of data. In particular, it is necessary to reassure that erroneous data has filtered out, while the remaining one is consistent between sources, utilizes same units of measurement, number representation, that null values are handled properly etc. Hence, the ANN is trained on consistent data corresponding to the last year time-period.

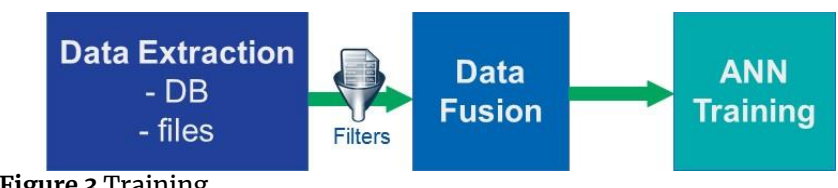

Figure 3 Training

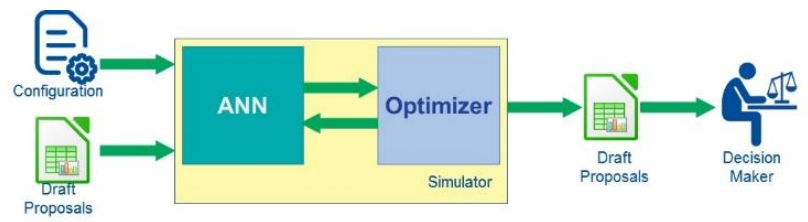

Figure 4 Solution search
In order to facilitate development and deployment of the system, it was decided to employ well-documented and supported Python libraries for AI: Tensorflow and Keras (Géron, 2019). In the same time, such libraries as Pandas take care on extraction of data from the input files (McKinney, 2012).

\section{Results and Discussion}

The authors conducted multiple tests on available data in order to evaluate efficiency of the solution. In particular, it was found that error in forecasting of sales is quite low and in $50 \%$ of the cases is lower than $20 \%$ of expected sales value. As was anticipated, the system does not provide a 'preset' solution considered the best and immediately employed. Instead, it generates a small set of proposals that the decision makers could accept, modify manually or discard completely. Indeed, despite technical possibility to create a fully autonomous system, the goal of this project is to operate with human-in-the-loop and to facilitate routine operations, saving precious time of the qualified personnel.

During the development, the program were continuously tested in collaboration with subject matter experts (SME) in order to secure sufficient level of precision and fine tune elaboration details. The system is currently in the final phase of development and is being subjected to performance and stress tests. In some cases, the real sales still could be different from forecasted ones. Indeed, manual analysis of some products revealed that even in apparently the same sales conditions the article could behave very differently. Regarding this issue, different hypothesis could be done: for instance, in some cases, data related to advertisement companies organized by producers and suppliers could be missing, hence in practice there could be a double advertisement company - one organized by chain of stores and other one by manufacturer. Obviously, such missing information could introduce significant errors on single products, however, this case seems to be quite exceptional and the total sales of all promoted products is expected to remain realistic

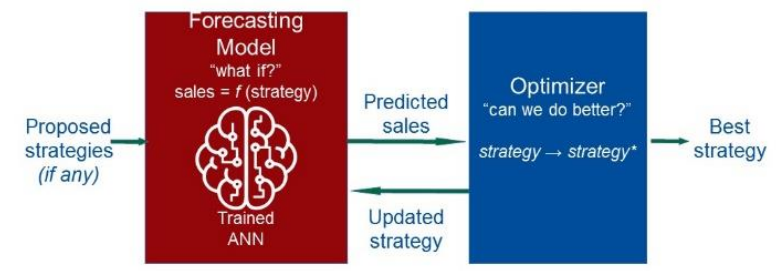

Figure 5 Forecasting model and optimizer

\section{Conclusions}

Nowadays it is possible to create complex forecasting and optimization systems using off-theshelf libraries and frameworks, freeing AI researchers from most of difficulties related to code writing. Indeed, this research proposes an innovative solution 
capable to support retail sales' decision makers in development of best advertising strategies with the aim to increase sales. The adoption of Strategic Engineering closed loop approach in combining AI, M\&S and Data Analytics results pretty promising and the authors are currently working on specific initiatives to carry out experimentations on real cases where to test their architecture and their innovative approach.

\section{References}

Ansari, M. E., \& Joloudar, S. Y. E. (2011). An investigation of TV advertisement effects on customers' purchasing and their satisfaction. International Journal of Marketing Studies, 3(4), 175.

d'Astous, A., \& Landreville, V. (2003). An experimental investigation of factors affecting consumers' perceptions of sales promotions. European Journal of Marketing.

Bogue, R. (2019). Strong prospects for robots in retail. Industrial Robot: the international journal of robotics research and application.

Brill, T. M., Munoz, L., \& Miller, R. J. (2019). Siri, Alexa, and other digital assistants: a study of customer satisfaction with artificial intelligence applications. Journal of Marketing Management, 35(15-16), pp. 1401-1436.

Bruzzone, A. G., Di Matteo, R., \& Sinelshchikov, K. (2018) Strategic Engineering \& Innovative Modeling Paradigms. In Workshop on Applied Modelling \& Simulation, Praha, Czech Republic, October 17-19

Bruzzone, A. G., \& Longo, F. (2010). An advanced system for supporting the decision process within largescale retail stores. Simulation, 86(12), 742-762.

Bruzzone, A. G., Bocca, E., \& Poggi, S. (2009). Renovating Intelligent Operations in Supermarket Chains. Proceedings of Third IEEE Asia International Conference on Modelling \& Simulation, Bandung, Indonesia, May, pp. 425-430

Bruzzone, A., Massei, M., \& Bocca, E. (2009). Freshfood supply chain. In Simulation-based case studies in logistics (pp. 127-146). Springer, London.

Bruzzone, A. G., Bocca, E., Pierfederici, L., \& Massei, M. (2009). Multilevel forecasting models in manufacturing systems. In Proceedings of Summer Computer Simulation Conference, Istanbul, Turkey, July, pp. 239-245

Bruzzone, A. G., Massei, M., \& Poggi, S. (2007). Simulation based analysis on different logistics solutions for fresh food supply chain. In Proceedings of Spring Simulation Multiconference, Norfolk, VA, March, Volume 3 (pp. 84-89).

Chung, M., Ko, E., Joung, H., \& Kim, S. J. (2018). Chatbot e-service and customer satisfaction regarding luxury brands. Journal of Business Research.
Darwin, C. (1859), On the Origin of Species by Means of Natural Selection, or the Preservation of Favoured Races in the Struggle for Life. London: John Murray, p. 502

Ferreira, K. J., Lee, B. H. A., \& Simchi-Levi, D. (2016). Analytics for an online retailer: Demand forecasting and price optimization. Manufacturing \& Service Operations Management, 18(1), pp. 69-88.

Géron, A. (2019). Hands-on machine learning with Scikit-Learn, Keras, and TensorFlow: Concepts, tools, and techniques to build intelligent systems. O'Reilly Media.

McKinney, W. (2012). Python for data analysis: Data wrangling with Pandas, NumPy, and IPython. " O'Reilly Media, Inc.".

Pak, O., Ferguson, M., Perdikaki, O., \&Wu, S. M. (2020). Optimizing stock-keeping unit selection for promotional display space at grocery retailers. Journal of Operations Management, 66(5), 501-533.

Kengpol, A., \& Wangananon, W. (2006). The expert system for assessing customer satisfaction on fragrance notes: Using artificial neural networks. Computers \& Industrial Engineering, 51(4), pp. 567584 .

Kumar, S., \& Zymbler, M. (2019). A machine learning approach to analyze customer satisfaction from airline tweets. Journal of Big Data, 6(1), 62.

Setiabudi, D. H., \& Tjahyana, L. J. (2011). Mobile Phone as a Personal Digital Shopping Assistant to Help Customers Shop in Shopping Center. International Journal of Information and Education Technology, 1(2), pp. 126-131.

Verstraete, G., Aghezzaf, E. H., \& Desmet, B. (2019). A data-driven framework for predicting weather impact on high-volume low-margin retail products. Journal of Retailing and Consumer Services, 48, 169-177.

Wallace, M., Maglogiannis, I., Karpouzis, K., Kormentzas, G., \& Kollias, S. (2003). Intelligent one-stop-shop travel recommendations using an adaptive neural network and clustering of history. Information Technology \& Tourism, 6(3), pp. 181193. 